\title{
Adeno-associated virus serotype 9 mediated vascular endothelial growth factor gene overexpression in mdx mice
}

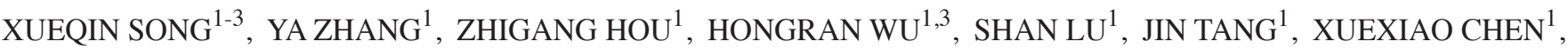 \\ HONGYING CUI ${ }^{1}$, YUAN LI ${ }^{1}$, YUE BI ${ }^{1,3}$, WEISONG DUAN ${ }^{3}$, ZHONGYAO LI $^{3}$ and CHUNYAN LI ${ }^{1-3}$ \\ ${ }^{1}$ Department of Neurology, The Second Hospital of Hebei Medical University; ${ }^{2}$ Institute of Cardiocerebrovascular Disease; \\ ${ }^{3}$ Neurological Laboratory of Hebei, Shijiazhuang, Hebei 050000, P.R. China
}

Received September 9, 2016; Accepted September 13, 2017

DOI: $10.3892 / \mathrm{etm} .2017 .5610$

\begin{abstract}
Duchenne muscular dystrophy (DMD) is a fatal neuromuscular disease caused by the absence of dystrophin. Vascular endothelial growth factor (VEGF) is a heparin-binding dimeric glycoprotein and principal angiogenic factor stimulating the migration, proliferation and expression of various genes in endothelial cells. Recently, VEGF was demonstrated to exhibit an antiapoptotic and direct myogenic effect, as well as to enhance muscle force restoration subsequent to traumatic injury. Therefore, the present study attempted to assess the muscle damage of VEGF overexpression in mdx mice. Adeno-associated virus serotype 9 (AAV9)-VEGF was administered intravenously to mdx mice. At 4 weeks after injection, VEGF was observed to be upregulated in the tibialis anterior muscle. In addition, the serum creatine kinase levels were significantly reduced and fatigue was slowed down, whereas the limb grip strength and weight of mice were markedly increased compared with the saline-treated mdx mice. Furthermore, significantly reduced inflammation and necrosis areas were observed in the muscle tissues of mice in the AAV9-VEGF group. These results suggested that AAV9-mediated VEGF gene overexpression was able to improve the muscle damage in mdx mice.
\end{abstract}

\section{Introduction}

Duchenne muscular dystrophy (DMD) is one of the most common and severe $\mathrm{X}$-linked recessive diseases, caused by mutations in the dystrophin gene. Current data suggest that DMD affects $\sim 1$ in 5,000 newborn males, it mainly effects males as it is an $\mathrm{X}$ linked recessive disease (1). The disease

Correspondence to: Dr Chunyan Li, Department of Neurology, The Second Hospital of Hebei Medical University, 215 West Heping Road, Shijiazhuang, Hebei 050000, P.R. China

E-mail: hebeichunyanli@aliyun.com

Key words: duchenne muscular dystrophy, adeno-associated virus 9, vascular endothelial growth factor, mdx mice, muscle pathology, muscle force, creatine kinase is characterized by progressive weakness of the skeletal and cardiac muscles, resulting in loss of ambulation and mortality due to cardiac or respiratory failure in teenagers (2).

The most widely studied animal model of DMD is the mdx mouse, whose dystrophy gene has a point mutation in exon 23 that causes the gene to terminate prematurely and does not encode a complete gene product, resulting in a defective dystrophin (3). Dystrophin is a key component of the dystrophy glycoprotein complex, which links the cytoskeleton of the muscle fibers with the extracellular matrix (4). Absence of dystrophin damages the sarcolemmal integrity and causes myofiber contraction-induced injury. It also inhibits signal transduction, including mechanical force transmission and cellular signal transduction. Previous studies have demonstrated that loss of dystrophin not only resulted in more fragile muscle cells, but also led to secondary vasodilatory dysfunction and the reduction of vascularization in the muscle tissue, consequently causing tissue ischemia (5-8).

Vascular endothelial growth factor (VEGF) is a main regulator of physiological and pathological angiogenesis. It increases vascular permeability, as well as induces the proliferation and migration of endothelial cells and new vascular structures (9). VEGF also promotes the production of NO and prostacyclin by endothelial cells, causing vasodilation. Verma et al (10) observed that enhanced vasculature was able to increase muscle satellite cells. Following ischemic muscle damage caused by glycerol or cardiotoxin, overexpression of VEGF markedly reduced the muscle damage and promoted muscle fiber regeneration (11). Angiotensin converting enzyme inhibitors have also been demonstrated to improve cardiac function and systemic vasodilator capacity in DMD patients (12). Therefore, vascular targeting therapy is considered as an effective strategy for DMD.

VEGF is known to be involved in angiogenesis, muscle cell regeneration and perfusion $(9,13)$. One of the promising vector systems for muscle gene transfer is adeno-associated virus (AAV). Among the different AAV serotypes, AAV serotype 9 (AAV9) vectors efficiently transduce skeletal and cardiac muscle following systemic administration (14). Thus, in the present study, a recombinant AAV vector was used to overexpress VEGF-165 in $\mathrm{mdx}$ mice, with the aim to investigate the effect of VEGF administration in the muscle pathology and function of this DMD mouse model. 


\section{Materials and methods}

Animal model. Specific-pathogen-free C57BL/10ScSn$\mathrm{Dmd}^{\mathrm{mdx}} / \mathrm{Nju}$ mice (referred to as $\mathrm{mdx}$ mice) and C57BL/10ScSn mice (referred to as wild-type (WT) mice) were purchased from Nanjing University Model Animal Research (Nanjing, China). The mice were split into groups as follows: A WT group $(\mathrm{n}=5$; weight, 24.7-29.7 $\mathrm{g}$; average weight, $27.0 \mathrm{~g}$ ), an $\mathrm{mdx}+\mathrm{NS}$ group ( $\mathrm{n}=5$; weight, 28.2-32.5 g; average weight, $29.9 \mathrm{~g}$ ) and an mdx+AAV9-VEGF group $(\mathrm{n}=5$; weight, 24.4-30.6; average weight, $28.5 \mathrm{~g})$. All animals were kept in a clean environment with a 12-h light and 12-h dark cycle at $22-25^{\circ} \mathrm{C}$ and $50-60 \%$ humidity, fed with sterile rodent granular feed and had free access to water and food. The study protocol was approved by the Ethics Review Board of the Second Hospital of Heibei Medical University (Shijiazhuang, China). All of the procedures were conducted in accordance with the Declaration of Helsinki 2016 and medical ethics policy in China (15).

Production of recombinant AAV9-VEGF-165 (AAV9-VEGF) vectors. VEGF-165 was transfected into the pseudotyped AAV9 vectors from the 293 cell line (Beijing Fiveplus Molecular Medicine Institute, Beijing, China) according to a previously published protocol (16). Pseudotyped AAV9 vectors were generated by packaging AAV2-based recombinant sc genomes into AAV9 capsids. The vectors were produced by helper virus-free three-plasmid transfection in 293 cells, using i) the adenovirus helper plasmid, ii) the AAV packaging plasmid encoding the rep2 and cap 9 genes (p5E18-VD2/9 for AAV9); and iii) the gene encoding murine VEGF (in fusion with a c-Myc tag) under control of the phosphoglycerate kinase promoter. The sc genome containing plasmids were constructed by deleting the $\mathrm{D}$ sequence and the terminal resolution site from one of the inverted terminal repeats. Recombinant vectors (rAAV) were purified by double- $\mathrm{CsCl}$ ultracentrifugation followed by dialysis against phosphate-buffered saline (five buffer changes, $3 \mathrm{~h}$ per round of dialysis) according to a previously published protocol (16). Physical particles were quantified by real-time PCR for vectors injected into mice and by dot blot hybridization for vectors injected into mice at 90 days of age. Vector titers are expressed as viral genomes per milliliter $(\mathrm{vg} / \mathrm{ml})$. Subsequent to purification and dialysis, the viral vector were stored at $-80^{\circ} \mathrm{C}$. Polymerase chain reaction (PCR) assay was performed in a $20 \mu 1$ system $(10 \mu 1$ SYBR-Green, $2 \mu 1$ forward primer, $2 \mu \mathrm{l}$ reverse primer, $2 \mu \mathrm{l}$ sample, $4 \mu \mathrm{lddH} 2 \mathrm{O}$ ). After holding the samples at $95^{\circ} \mathrm{C}$ for $10 \mathrm{~min}, 40$ cycles of amplification at $95^{\circ} \mathrm{C}$ for $10 \mathrm{sec}, 60^{\circ} \mathrm{C}$ for $20 \mathrm{sec}$ and $72^{\circ} \mathrm{C}$ for $15 \mathrm{sec}$ was performed. Synthetic primers and SYBR-Green (cat no. GK8020; Generay Biotech Co., Ltd., Shanghai, China) were used for quantitative PCR on an M3005P (Agilent Technologies, Inc., Santa Clara, CA, USA). The following primer sequences were used: Forward, 5'-CCATCCTGT GTGCCCCTGATGC-3' and reverse 5'-TCCTCTCTACTC GAAGGATGTC-3.

Animal grouping and administration. Male C57BL/10 mice (6-week-old) were selected as the WT group. The male mdx mice (6-week-old) were selected for use in the experiments and divided into two groups, as follows: mdx+AAV9-VEGF group $(\mathrm{n}=5), 150 \mu \mathrm{l}$ AAV9-VEGF was injected into each mouse via the tail vein once; and the $\mathrm{mdx}+$ normal saline (NS) group $(n=5)$, administered with the same amount of NS via the tail vein once. The degree of muscle damage was quantitatively assessed after 4 weeks, when the mice were 10-weeks-old.

Forelimb grip strength test. In order to assess the mouse forelimb strength, a Grip Strength Meter (Columbus Instruments International, Columbus, $\mathrm{OH}, \mathrm{USA}$ ) was used. Briefly, C57BL/10 and mdx mice were lifted over the steel grid plate by the tail in order to grasp the grid plate with their forelimbs. Next, the animals were pulled back by the tail until their forelimbs were released from the grid plate. During the measurement, the tension was not severe or slow, and the hind legs did not touch the grid plate. All mice were tested on the same day by the same researcher. The grip strength of each mouse was examined five times and recorded in terms of gram-force units. The three highest measured values were averaged to calculate the mean grip strength, which was normalized against the body weight $(\mathrm{g})$ of the mice. Each mouse was subjected to the test for five times with 1-min interval between tests. The grip strength of the mdx group was compared with that of the control group. The fatigue refers to the decline in the percentage of grip strength that was calculated by comparing the first and the fifth grip strength examination.

Collecting serum samples. Mice were sacrificed using $10 \%$ chloral hydrate (Tianjin Yongda Chemical Reagent Co., Ltd., Tianjin, China) via intraperitoneal injection. Subsequently, the eyeballs were removed, and a blood sample of $\sim 1.0 \mathrm{ml}$ was collected. After standing $2 \mathrm{~h}$ at room temperature, the samples were kept at $4^{\circ} \mathrm{C}$ refrigerator for $15 \mathrm{~min}$ and were then, centrifuged at $956 \mathrm{xg}$ for $10 \mathrm{~min}$ at $4^{\circ} \mathrm{C}$, and the supernatant was collected and stored at $-80^{\circ} \mathrm{C}$. Serum creatine kinase (CK) was measured by an indirect CK colorimetric assay kit (Beijing Leadman Biochemistry Co., Ltd., Beijing, China).

Pathological staining. Following sacrifice, the gastrocnemius muscle were removed and stored at $-80^{\circ} \mathrm{C}$. Tragacanth gum was mixed with water and kept for $30 \mathrm{~min}$ at $20-25^{\circ} \mathrm{C}$, prior to being loaded into a pyramid. The volume of sample was less than half the inside of the pyramid. The transection of myofiber was kept upward. The isopentane was maintained at freezing point $\left(-155-160^{\circ} \mathrm{C}\right)$. The pyramid was placed into isopentane and stirred rapidly for $\sim 30 \mathrm{sec}$. Then, samples were stored at $-80^{\circ} \mathrm{C}$. Samples were sliced into $8-\mu \mathrm{m}$ thick sections. The frozen sections were stained with hematoxylin and eosin (H\&E; Sinopharm Chemical Reagent Co., Ltd., Shanghai, China).

Quantitative evaluation of histological parameters. An image acquisition system (Olympus BX51; Olympus Corporation, Tokyo, Japan) was used to examine at least 10 fields-of-view (magnification, $\mathrm{x} 400$ ) in each mouse. The proportion of core fibers (or regeneration of muscle fibers) over the total number of muscle fibers in the tissue sections was calculated. Core fibers were identified using the following criteria: Normal mature fiber size; and nucleus in the central position. Muscle 
fiber regeneration was identified using the following criteria: Smaller cell size than the normal mature muscle fibers; nucleus in a central location; and basophilic or non-basophilic cytoplasm.

Using an image analysis system (Image-Pro Plus version 6; Media Cybernetics, Inc., Rockville, MD, USA), the central core fibers (or regenerated muscle fibers) and total number of muscle fibers were counted.

Using Image J software 1.48v (National Institutes of Health, Bethesda, MD, USA), at x40 magnification, a complete lateral section image of the muscle tissue was obtained and at x200 magnification, images of all inflammatory necrosis areas were obtained. The percentage of the inflammatory necrosis area over the total area was then calculated. Analysis was conducted by neurology professionals.

Western blotting. After the mice were sacrificed and the tibialis anterior muscle was collected, lysate protein was extracted from the tissues using protein extraction kit P1250 (Applygen Technologies Inc., Beijing, China). The Bradford method was used for protein quantitation, and then $50-\mu \mathrm{g}$ protein samples were separated by $8-12 \%$ SDS-PAGE. Next, the proteins on the gel were transferred onto polyvinylidene difluoride membranes (EMD Millipore, Billerica, MA, USA). The membranes were then incubated overnight at $4^{\circ} \mathrm{C}$ with primary antibodies against VEGF (1:500; ab46154; Abcam, Cambridge, MA, USA) and actin (1:500; cat no. TA-09; Beijing Zhongshan Golden Bridge Biotechnology Co., Ltd., Beijing, China), serving as an internal control. Subsequently, the membranes were stained with anti-rabbit Immunoglobin $\mathrm{G}$ peroxidase conjugated secondary antibody (1:10,000; cat no. 611-1302; Rockland Immunochemicals Inc. Pennsylvania, USA) for $1 \mathrm{~h}$ at room temperature. An Odyssey infrared imaging system 1.2 (LI-COR, Lincoln, NE, USA) was used to detect the bands of interest. Data were analyzed using GraphPad Prism 4.0 (GraphPad Software, Inc., La Jolla, CA, USA) and compared by F-test.

Statistical analysis. The data were analyzed using SPSS version 13.0 software (SPSS, Inc., Chicago, IL, USA). All values are expressed as the mean \pm standard deviation or as the median (interquartile range). Differences between groups were compared with single-factor analysis of variance followed by Student Newman Keuls test or Fisher's Least Significant Difference, while median values were compared with multiple independent-samples nonparametric test. $\mathrm{P}<0.05$ was considered to indicate differences that were statistically significant.

\section{Results}

VEGF expression is increased in AAV9-VEGF-treated $m d x$ mice. According to the western blotting results, the VEGF content in the tibialis anterior muscle of the AAV9-VEGF-treated mdx mice was significantly increased compared with that in the NS-treated mdx and the wild-type (WT) mice (Fig. 1). This indicated that VEGF overexpression was achieved in the AAV9-VEGF group.

Serum CK level is reduced by AAV9-VEGF treatment in mdx mice. Serum CK is one of the commonly used indicators for muscle damage (17). A low serum CK level was observed in
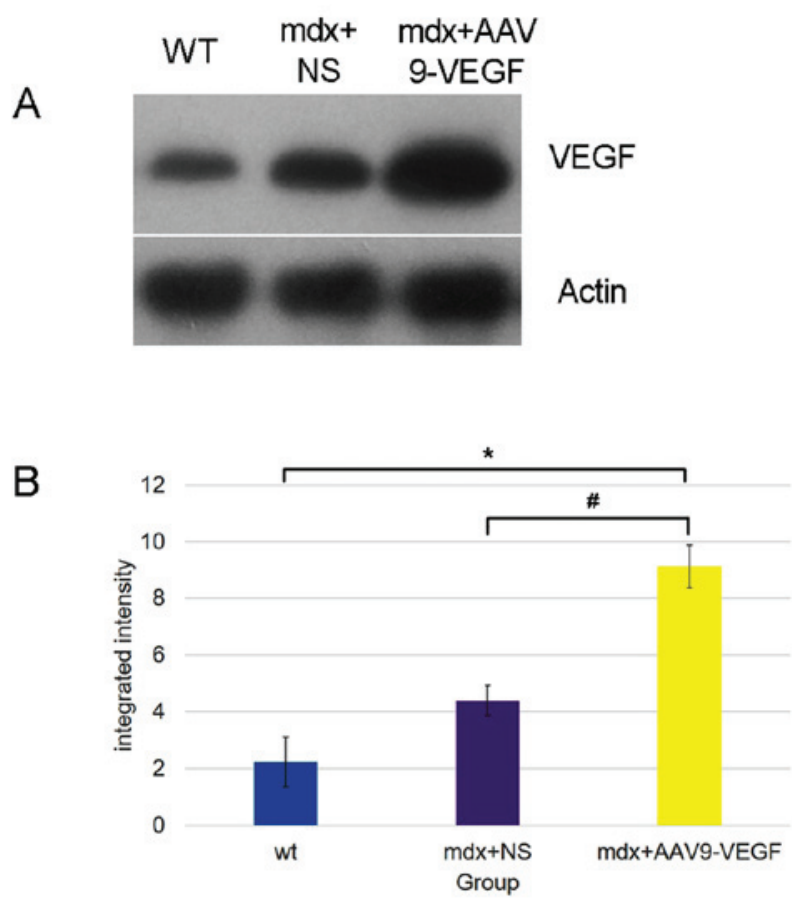

Figure 1. Western blot analysis of VEGF protein level in the tibialis anterior muscle tissue of WT, NS-treated mdx and AAV9-VEGF-treated mice. (A) Increased VEGF expression in AAV9 VEGF-treated mdx mice. (B) VEGF semi-quantification revealed a $~ 3.3$-fold increase in AAV9 VEGF-treated muscle compared with WT $(\mathrm{n}=3 ; \mathrm{P}=0.001)$ and $\sim 1.8$-fold increase in AAV9 VEGF-treated muscle compared with NS-treated muscle $(n=3 ; P=0.007)$ ${ }^{*} \mathrm{P}<0.05$ vs. WT group; ${ }^{\#} \mathrm{P}<0.05$ vs. $\mathrm{mdx}+\mathrm{NS}$ group. VEGF, vascular endothelial growth factor; AAV9, adeno-associated virus serotype 9; NS, normal saline; WT, wild-type.

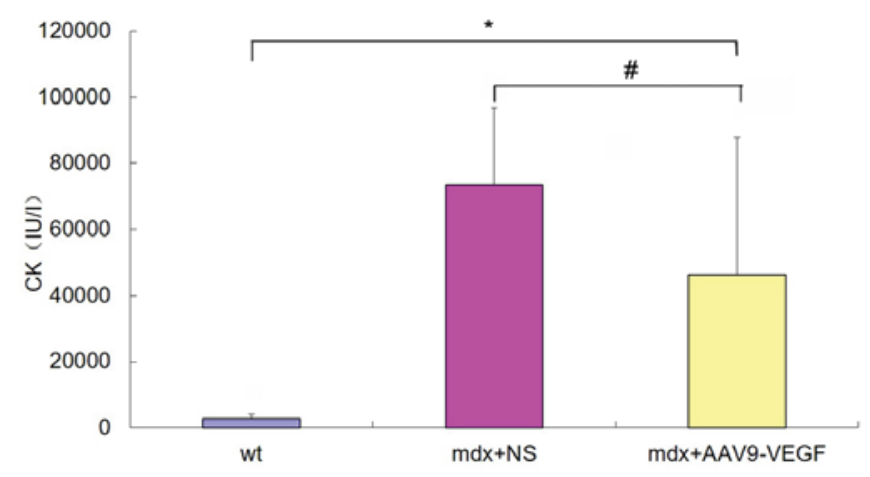

Figure 2. Serum CK levels in the WT, $m d x+$ NS and mdx + AAV9-VEGF mice at 10 weeks of age. Data is presented as the median and interquartile range. ${ }^{*} \mathrm{P}<0.05 \mathrm{mdx}+\mathrm{AAV} 9-\mathrm{VEGF}$ group vs. WT group; ${ }^{*} \mathrm{P}<0.05$ $\mathrm{mdx}+\mathrm{AAV9-VEGF}$ group vs. $\mathrm{mdx}+\mathrm{NS}$ group. CK, creatine kinase; VEGF, vascular endothelial growth factor; AAV9, adeno-associated virus serotype 9; NS, normal saline; WT, wild-type.

the WT group (2,746.5 U/1), while the CK level was significantly increased in NS-treated mdx mice $(73,418 \mathrm{U} / 1 ; \mathrm{P}<0.05)$. In mdx mice treated with AAV9-VEGF, the serum CK level (46,249.5 U/1) was significantly higher in comparison with that of the WT group, while it was significantly lower compared with that in NS-treated mdx mice $(\mathrm{P}<0.05$; Fig. 2).

Grip strength of mice. The results indicated significantly higher forelimb strength in the AAV9-VEGF-treated mdx mice 


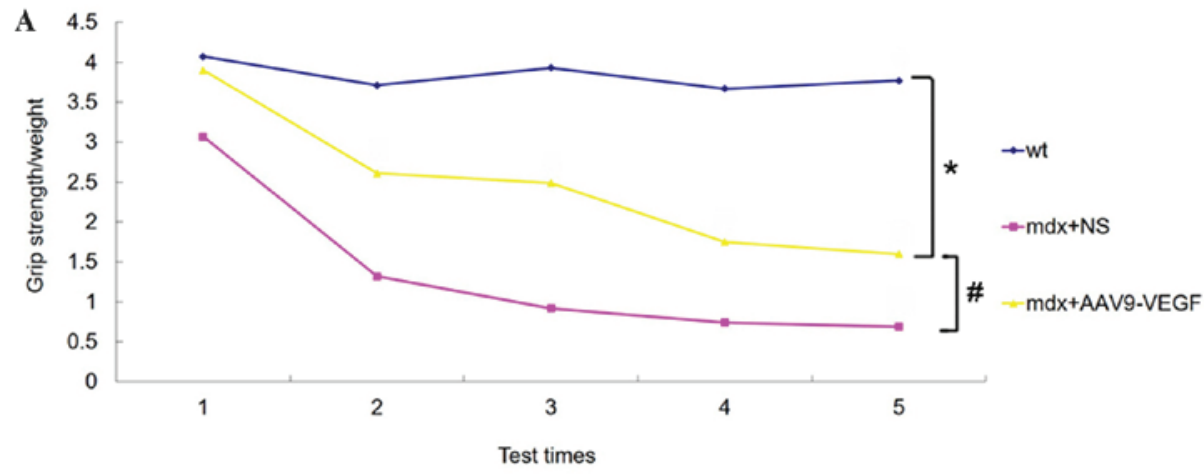

B

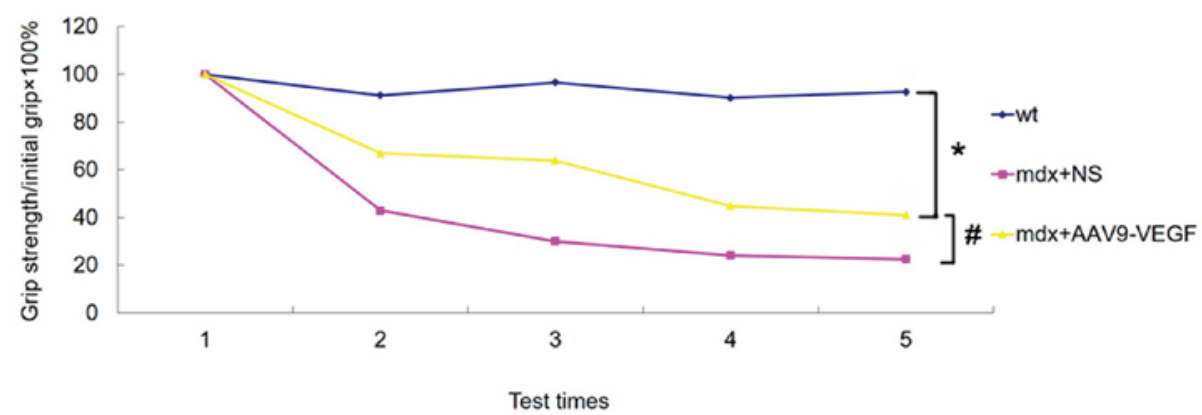

Figure 3. AAV9-mediated VEGF gene overexpression improved the grip strength in mdx mice. (A) Forelimb grip strength normalized to the weight, and (B) the decrease in forelimb grip force in terms of the initial grip. ${ }^{\mathrm{P}}<0.05 \mathrm{mdx}+\mathrm{NS}$ group vs. WT group; ${ }^{*} \mathrm{P}<0.05 \mathrm{mdx}+\mathrm{AAV} 9-\mathrm{VEGF}$ group vs. mdx $+\mathrm{NS}$ group. VEGF, vascular endothelial growth factor; AAV9, adeno-associated virus serotype 9; NS, normal saline; WT, wild-type.

when compared with the NS-treated mdx mice, although the strength remained lower than that in the WT group (Fig. 3A and Table I). Between the first and fifth round of the test, the level of fatigue in the AAV9-VEGF, NS and WT groups was $\sim 57,77$ and 6\%, respectively (Fig. 3B). It indicated that VEGF could improve the muscle function at some degree.

Histopathological study. According to the results of H\&E staining, the muscle tissue pathological alterations observed in mdx mice mainly included muscle fiber hypertrophy and atrophy, muscle fiber necrosis, inflammatory cell infiltration and muscle cell regeneration, as compared with the WT mice. Muscle fiber necrosis and inflammatory cell infiltration in AVV9-VEGF-treated mdx mice was reduced markedly compared with the NS-treated mdx mice (Fig. 4). Muscle regeneration and muscle necrosis was observed in both the AAV9-VEGF-treated mdx mice and NS-treated mdx mice.

The proportion of inflammatory and necrosis area in the three groups of mice was calculated, and a statistically significant difference was detected among the three groups $(\mathrm{F}=0.957, \mathrm{P}=0.015)$. More specifically, the proportion of inflammatory and necrosis area in the AAV9-VEGF group $(0.992 \pm 0.44 \%)$ was significantly reduced when compared with that of the NS group $(13.01 \pm 9.5 \%)(\mathrm{P}=0.013)$ and WT group; Fig. 5A). This indicated that AAV9-mediated VEGF gene overexpression reduced the muscle inflammation and necrosis in the mdx mouse. Regarding muscle regeneration, the percentage of regenerated muscle fibers in the AAV9-VEGF group was $27.97 \pm 7.37 \%$, which was higher in comparison with that in the NS group $(18.39 \pm 7.16 \%)$; however, this difference was not statistically significant (P>0.05; Fig. 5B). These findings suggested that VEGF significantly decreases inflammation and necrosis in the muscle of mdx mice, whereas the effect of VEGF on the regeneration of muscle cells may not be marked under this administration.

\section{Discussion}

VEGF is a key regulator of vascularization and pathological angiogenesis, including in tumors and intraocular neovascularization disease (18). VEGF promotes angiogenesis through vascular and endothelial progenitor cells, while it also promotes vascularization by migration of endothelial cells. In addition, VEGF overexpression may be used to treat ischemic tissues, since this factor serves a key role in vascularization and blood flow in skeletal muscles. Knockdown of VEGF in the skeletal muscle of adult mice leads to a 2-fold increase in capillary distribution in muscle tissue, a $\sim 80 \%$ decrease in the activity tolerance time, and elimination of vascularization (19). The combination of AAV and VEGF treatment has been applied in different models of muscle necrosis to increase vascular density, protect the muscle cells and promote regeneration (11). VEGF was initially considered to be an endothelial specific growth factor; however, recent studies demonstrated that VEGF also promoted the regeneration of numerous other cells, including neurons, hepatocytes, osteoblasts, hematopoietic cells and muscle cells (20). In the present study, the effect of VEGF on muscle 
Table I. Forelimb grip strength normalized to the weight of mice in the WT, NS and AAV9-VEGF groups at 10 weeks of age.

\begin{tabular}{|c|c|c|c|c|c|}
\hline Test round & $\mathrm{n}$ & WT & $\mathrm{mdx}+\mathrm{NS}$ & AAV9-VEGF & P-value \\
\hline 1 & 5 & $4.07 \pm 0.70$ & $3.07 \pm 0.60$ & $3.91 \pm 0.40$ & 0.027 \\
\hline 2 & 5 & $3.71 \pm 0.35$ & $1.32 \pm 0.58$ & $2.61 \pm 0.82$ & 0.001 \\
\hline 3 & 5 & $3.93 \pm 0.35$ & $0.92 \pm 0.29$ & $2.49 \pm 0.61$ & 0.000 \\
\hline 4 & 5 & $3.67 \pm 0.54$ & $0.74 \pm 0.24$ & $1.75 \pm 0.56$ & 0.004 \\
\hline 5 & 5 & $3.77 \pm 0.47$ & $0.69 \pm 0.21$ & $1.60 \pm 0.47$ & 0.003 \\
\hline
\end{tabular}

WT, wild-type; NS, normal saline; VEGF, vascular endothelial growth factor; AAV9, adeno-associated virus serotype 9.
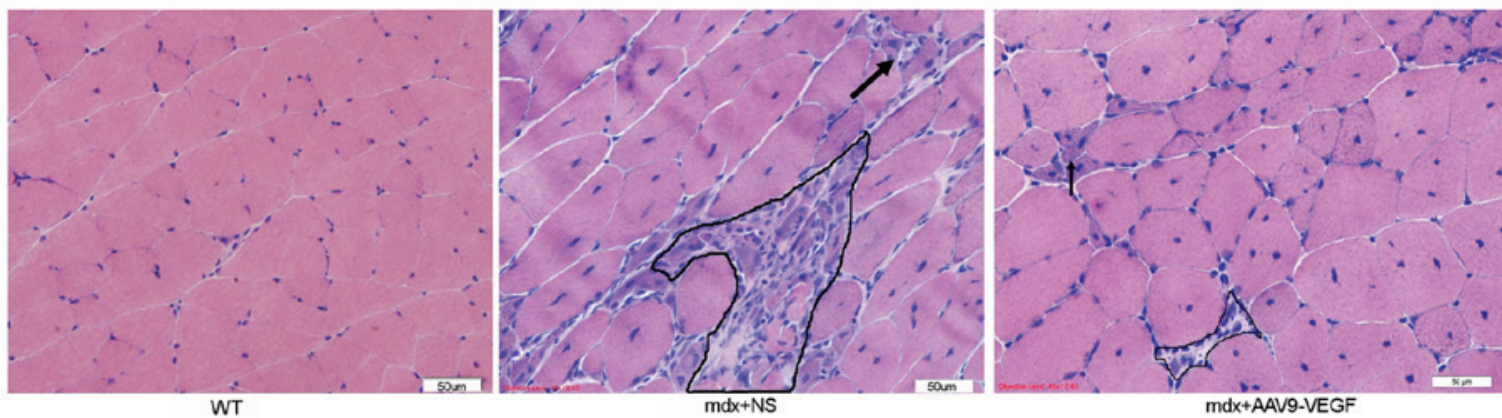

Figure 4. Pathological alterations in the gastrocnemius muscle of 10-week-old mdx mice compared with the 10-week-old C57BL/10 mice (controls). The black line area demonstrates the necrosis, where the cell membrane appears to be incomplete, with inflammatory cell infiltration, pale cytoplasm and phagocytosis observed. Regeneration of the cells is shown by the black arrow, with small size, central nuclei with basophilic cytoplasm. In AVV9-VEGF mice, muscle fiber hypertrophy muscle fiber necrosis, inflammatory cell infiltration and muscle cell regeneration were observed in muscle tissue. Hematoxylin and eosin staining; magnification, x400. VEGF, vascular endothelial growth factor; AAV9, adeno-associated virus serotype 9; NS, normal saline; WT, wild-type.
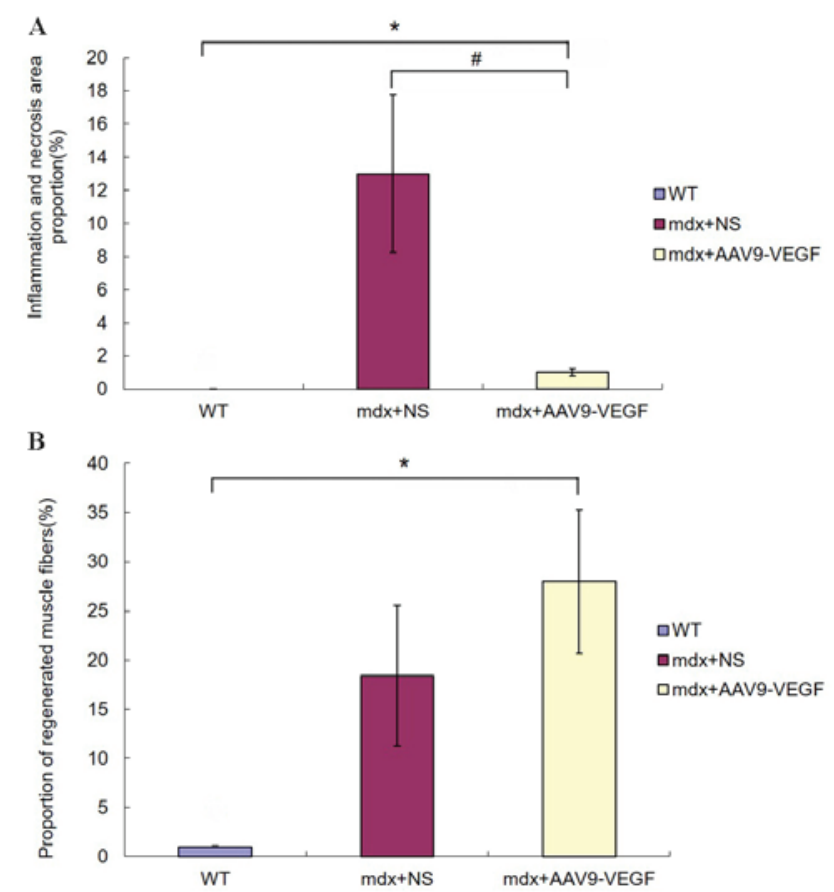

Figure 5. AAV9-mediated VEGF gene overexpression improved the muscle pathology in mdx mice. (A) The percentage of the inflammatory necrosis area determined in the muscle tissue was decreased in AAV9-VEGF-treated mice. (B) The percentage of regeneration muscle fibers was increased in the muscle tissues of AAV9-VEGF-treated mice, although the difference was not statistically significant compared with the $\mathrm{mdx}+$ NS mice. ${ }^{*} \mathrm{P}<0.05$ mdx + AAV9-VEGF group vs. WT group; ${ }^{*} \mathrm{P}<0.05 \mathrm{mdx}+$ AAV9-VEGF group vs. mdx + NS group. VEGF, vascular endothelial growth factor; AAV9, adeno-associated virus serotype 9; NS, normal saline; WT, wild-type. pathology and muscle strength was further explored in mdx mice, serving as a DMD animal model. The current study was based on systemic administration of AAV9-VEGF by tail vein injection, which is a more uniform and suitable for the clinical treatment of systemic diseases. The AAV9 vector has a high affinity with muscle tissue, and can reduce the effect of drugs on other tissues and organs. Compared with other AAV serotypes, AAV9 has a slower clearance rate and carries the gene expression in the body for long periods of time (21).

In the present study, the quantitative muscle damage in AAV9-VEGF-treated mdx mice was compared with that in NS-treated mdx mice. In terms of the serological index, serum CK in the AAV9-VEGF group was evidently lower in comparison with the NS group, but higher compared with the WT group. Regarding muscle strength, the AAV9-VEGF and NS mdx groups presented a markedly decreased strength compared with the WT group. However, the AAV9-VEGF group demonstrated significant improvement compared with the NS group. In the limp grip strength test, the first round was considered as the $100 \%$ baseline. The grip strength of the AAV9-VEGF group was significantly decreased 57\%, whereas it was reduced $77 \%$ in the NS-treated mdx group; therefore, this indicated that VEGF improved the muscle function of mdx mice. Furthermore, according to the histopathological analysis, the AAV9-VEGF group exhibited evident necrosis, although the area of inflammation necrosis was significantly reduced when compared with the NS treated mice. These results indicated that VEGF improved the muscle function of 
mdx mice. It was also suggested that VEGF overexpression may improve the muscle tissue ischemia anoxic condition by increasing the vascular density in mdx mice, thus reducing the muscle fiber necrosis. Similar observations were obtained by immunohistochemical staining of vascular density alterations and quantitative PCR. Compared with the mdx group, the proportion of muscle fiber regeneration in the AAV9-VEGF group slightly increased without a statistically significant difference. The possible reasons underlying this effect may be the following: Firstly, overexpression of VEGF decreased the necrosis of the muscle tissue in mdx mice. Thus, the proportion of degenerative fibers was used as an indicator of the pathology of muscular tissue degeneration and regeneration in the mdx mice. By contrast, through the reduction of muscle tissue necrosis, regenerative fibers may be correspondingly reduced. In addition, previous in vitro and in vivo experiments demonstrated that VEGF directly promoted muscle regeneration, and this promoting effect was dose-dependent (20). In the present study, the dosage of VEGF may have been unable to promote sufficient muscle regeneration. Furthermore, the mdx mice had a strong regeneration ability at the examined time points, which may reduce the effect of VEGF on muscle regeneration. In weeks 4-12, the muscle of mdx mice regenerated to some extent. The pathological alterations were stabilized and necrosis was reduced after 12 weeks.

In future work, the dose of VEGF should be increased to study the effect of VEGF on blood vessel formation and maturation. However, an excessive accumulation in the local tissues may lead to blood vessel growth disorder, which does not increase blood perfusion, but can cause tissue ischemia (22). Thus, it is crucial to further examine the appropriate VEGF dose.

In conclusion, VEGF has been widely used to investigate muscle tissue-associated diseases, although only a limited number of studies exist on DMD. In the present study, it was confirmed that VEGF decreases serum CK levels, increases grip strength, slows down fatigue in mdx mice; and significantly decreases inflammation and necrosis in the muscle of mdx mice, whereas the effect of VEGF on the regeneration of muscle cells may not be significant. However, further investigation into the specific mechanisms, signal transduction pathways and benefits of long-term treatment is required.

\section{References}

1. Mendell JR, Shilling C, Leslie ND, Flanigan KM, al-Dahhak R, Gastier-Foster J, Kneile K, Dunn DM, Duval B, Aoyagi A, et al: Evidence-based path to newborn screening for duchenne muscular dystrophy. Ann Neurol 71: 304-313, 2012.

2. Emery AEH and Muntoni F: Duchenne Muscular Dystrophy. 3rd edition. Oxford University Press, Oxford, NY, p270, 2003.

3. Sicinski P, Geng Y, Ryder-Cook AS, Barnard EA, Darlison MG and Barnard PJ: The molecular basis of muscular dystrophy in the mdx mouse: A point mutation. Science 244 $1578-1580,1989$.
4. Ibraghimov-Beskrovnaya O, Ervasti JM, Leveille CJ, Slaughter CA, Sernett SW and Campbell KP: Primary structure of dystrophin-associated glycoproteins linking dystrophin to the extracellular matrix. Nature 355: 696-702, 1992.

5. Davies KE and Nowak KJ: Molecular mechanisms of muscular dystrophies: Old and new players. Nat Rev Mol Cell Biol 7: 762-773, 2006.

6. Brenman JE, Chao DS, Xia H, Aldape K and Bredt DS: Nitric oxide synthase complexed with dystrophin and absent from skeletal muscle sarcolemma in Duchenne muscular dystrophy. Cell 82: 743-752, 1995.

7. Loufrani L, Matrougui K, Gorny D, Duriez M, Blanc I, Lévy BI and Henrion D: Flow (shear stress)-induced endothelium-dependent dilation is altered in mice lacking the gene encoding for dystrophin. Circulation 103: 864-870, 2001.

8. Davies PF: Flow-mediated endothelial mechanotransduction. Physiol Rev 75: 519-560, 1995.

9. Ferrara N: Role of vascular endothelial growth factor in physiologic and pathologic angiogenesis: Therapeutic implications. Semin Oncol 29 (6 Suppl 16): S10-S14, 2002.

10. Verma M, Asakura Y, Hirai H, Watanabe S, Tastad C, Fong GH, Ema M, Call JA, Lowe DA and Asakura A: Flt-1 haploinsufficiency ameliorates muscular dystrophy phenotype by developmentally increased vasculature in mdx mice. Hum Mol Genet 19: 4145-4159, 2010.

11. Arsic N, Zacchigna S, Zentilin L, Ramirez-Correa G, Pattarini L, Salvi A, Sinagra G and Giacca M: Vascular endothelial growth factor stimulates skeletal muscle regeneration in vivo. Mol Ther 10: 844-854, 2004

12. Ennen JP, Verma M and Asakura A: Vascular-targeted therapies for duchenne muscular dystrophy. Skelet Muscle 3: 9, 2013.

13. Christov C, Chretien F, Abou-Khalil R, Bassez G, Vallet G, Authier FJ, Bassaglia Y, Shinin V, Tajbakhsh S, Chazaud B and Gherardi RK: Muscle satellite cells and endothelial cells: Close neighbors and privileged partners. Mol Biol Cel 18: 1397-1409, 2007.

14. Zincarelli C, Soltys S, Rengo G and Rabinowitz JE: Analysis of AAV serotypes 1-9 mediated gene expression and tropism in mice after systemic injection. Mol Ther 16: 1073-1080, 2008.

15. Nie JB: Medical Ethics in China. Routledge, London, 2011.

16. Wang Y, Duan W, Wang W, Di Wen, Liu Y, Liu Y, Li Z, Hu H, Lin $\mathrm{H}$, Cui C, et al: scAAV9-VEGF prolongs the survival of transgenic ALS mice by promoting activation of M2 microglia and the PI3K/Akt pathway. Brain Res 1648: 1-10, 2016.

17. Altamirano F, Perez CF, Liu M, Widrick J, Barton ER, Allen PD, Adams JA and Lopez JR: Whole body periodic acceleration is an effective therapy to ameliorate muscular dystrophy in $\mathrm{mdx}$ mice. PLoS One 9: e106590, 2014.

18. Cross MJ, Dixelius J, Matsumoto T and Claesson-Welsh L: VEGF-receptor signal transduction. Trends Biochem Sci 28: 488-494, 2003.

19. Crafts TD, Jensen AR, Blocher-Smith EC and Markel TA: Vascular endothelial growth factor: Therapeutic possibilities and challenges for the treatment of ischemia. Cytokine 71: 385-393, 2015.

20. Ferrara N, Gerber HP and LeCouter J: The biology of VEGF and its receptors. Nat Med 9: 669-676, 2003.

21. Zincarelli C, Soltys S, Rengo G and Rabinowitz JE: Analysis of AAV serotypes 1-9 mediated gene expression and tropism in mice after systemic injection. Mol Ther 16: 1073-1080, 2008.

22. Springer ML, Chen AS, Kraft PE, Bednarski M and Blau HM: VEGF gene delivery to muscle: potential role for vasculogenesis in adults. Mol Cell 2: 549-558, 1998.

This work is licensed under a Creative Commons Attribution-NonCommercial-NoDerivatives 4.0 International (CC BY-NC-ND 4.0) License. 